\title{
Electrical Characteristics of Transmission Systems
}

\author{
BY HERBERT BRISTOL DWIGHT \\ Me:nber, A. I. E. E. \\ Canadian Westinghouse Company, Lt\}.
}

\begin{abstract}
In making the electrical calculations for a long transmission line, it is desirable to include the effect of the step-up and step-down transformers and to make a direct calculation for the complete system, without any trial and error procedure. A method for doing this is described for constant-voltage lines, since long, high-power lines, especially those of 220,000 volts, usually require to be operated at constant voltage by means of synchronous condensers. The necessity of using the hy perbolic theory in calculating such lines is pointed out.
\end{abstract}

$\mathrm{T}$ HE electrical characteristics of a simple transmission line of uniform design throughout are usually calculated from the resistance, reactance and capacitance of the line. However, the characteristics of the line alone are often not so useful to know as the characteristics of the complete system, including transformers and synchronous condensers, and sometimes with different types of conductors used on different parts of the line. Where synchronous condensers are used, it is usual to assume that they hold the voltage constant at certain points.

In this article is shown a method of calculating the characteristics of a constant-voltage transmission system, including the effect of the transformers, the distributed capacitance of the line, and changes in size and grouping of conductors.

It may be stated as a well-established fact that any transmission line long enough, and with a power load large enough to justify the adoption of 220,000 volts, will require to be operated as a constant-voltage transmission line, using synchronous condensers.

The adoption of 220,000 volts means increased cost of transformers, circuit breakers, line insulators, and towers which must be large enough to provide wide spacing between conductors. It will therefore, be economical to use such a high voltage only for a large block of power transmitted a long distance, and this is found to require a low resistance conductor of large size, approximating one inch in diameter. This large size of conductor is required also in order to avoid trouble from corona, though a large diameter for this purpose may be secured by the expedient of using a large steel core. Now, overhead conductors of very large size have several times as much reactance as resistance, so that the maximum load which may be carried by the line is determined by voltage variation and not by line loss. It is in such cases that synchronous condensers for holding the line voltage constant have been found to be most pronitable.

It may also be stated that for any 220,000 -volt transmission system, and, indeed, for much less important systems, it is necessary to take accurate account of the distributed capacitance according to the hyperbolic theory, in order to avoid serious errors in the calculated results.

This is very well shown by the transmission line problem given in Fig. 5 of F. G. Baum's paper on

Presented at the Pacific Coast Convention of the A.I.E.E., Vancouver, B. C., August 8-11, 1922.
"Voltage Regulation and Insulation," Journal of the A. I. E. E., August, 1921, page 648. Mr. Baum used an approximate method of calculation which was not based on the hyperbolic theory. As a result, he obtained a value of $124,000 \mathrm{kv}-\mathrm{a}$. of synchronous condensers for a load of $104,000 \mathrm{kw}$. As a matter of fact, $82,000 \mathrm{kv}-\mathrm{a}$. of synchronous condensers are required for the line in question at full load, which is the only condition considered in Mr. Baum's paper. Considering both no-load and full-load conditions, the required capacity to maintain constant voltage is $244,000 \mathrm{kv}-\mathrm{a}$., or 235 per cent of the value of the load in kilowatts. There is also a considerable discrepancy in the calculated efficiency due to using the approximate method of calculation. The approximate method gives 71 per cent efficiency, but this should be 77 per cent, according to the data given.

It is doubtless true that synchronous condensers would be required at intervals in order to transmit power 800 miles at 220,000 volts, though possibly not at such close intervals as 150 miles. However, it is necessary to use the hyperbolic theory if even a rough estimate is to be made of the operation of the system or the amount of synchronous condensers required.

A very useful method of determining the size of conductor and the features of loading and controlling a constant-voltage transmission line, is to draw a circle diagram for the line in question. This shows the operation of the synchronous condensers under all conditions of load and it gives the maximum load which can be carried by the line at the voltages considered. The efficiency of transmission and power factor at the generators for various loads may also be conveniently plotted above the diagram. (See Fig. 2.)

The circle diagram is advantageous, first, because it gives results for all loads and not for one or two particular loads only. Second, because it is not a trial and error method but it gives results at once for the definite supply and receiver voltages chosen. In the third place, concentric circles may be drawn with practically no extra calculation whatever, to show the results for different values of the supply voltage $E_{s}$. (See Fig. 2.) In the fourth place, it is possible with very little extra work to obtain precise calculated results by means of the calculated data used in making the diagram.

The method of drawing a circle diagram of a constantvoltage transmission line, not including the step-up and step-down transformers, but taking account of the 
distributed capacity according to the hyperbolic theory, has been published by the author in "Constant-Voltage Transmission," pages 78 and 99. The present article gives formulas for drawing the diagram or calculating the results when the transformer resistances and reactances are included in the circuit, the notation used being similar to that in the book referred to. Average values have been taken for the transformer core loss and magnetizing current, and for the condenser loss, and these have been included in the calculation.

A similar method for including the transformer characteristics in the transmission circuit calculation has been worked up by Messrs. R. D. Evans and H. K. Sels and published by them in the Electric Journal. A useful reference in connection with this kind of calculation is "The Calculation of Transmission Line Networks" by Prof. T. R. Rosebrugh, Bulletin No. 1. 1919, of the School of Engineering Research, University of Toronto, which gives the general circuit constants for several lines in parallel, in series-parallel, and with intermediate loads, etc. Such general constants are often applicable in the following circle diagram calculation.

By making allowance for the transformer characteristics, the preliminary calculation is made somewhat longer than for the line alone, but the construction of the diagram itself is not made any more complicated in any way.

Let the constant voltage at the low-tension side of the receiving transformers be $E$ volts to neutral, (equivalent high-tension voltage). See Fig. 1. Let the load current combined with the reactive current from the synchronous condensers be $P+j Q$ amperes per conductor, (equivalent high-tension current). $Q$ is a positive quantity when leading and negative when lagging. Let the average loss in the synchronous condensers be represented by the current $P_{c}$, in phase with $E$. Let the core loss and magnetizing current of the receiving transformers be represented by the admittance $G_{t r}+j B_{t r}$ at the average operating high-tension voltage. Let the corresponding quantity for the supply transformers be $G_{t s}+j B_{t s}$. The core loss current and the magnetizing current of a given transformer are assumed to flow in the primary winding of that transformer and not in the secondary winding. The impedance of the receiving transformers is $R_{t r}+j X_{t r}$, and that of the supply transformers is $R_{t s}+j X_{t s}$.

The process of calculating the data for the circle diagram consists in starting at the load end, where the voltage $E$ and all other conditions are known except the current $P+j Q$. The voltage and current at each part of the system are then calculated, using numerical values of all quantities except that the letters $P+j Q$ will always appear. Thus finally the value of $E_{s}$ will be obtained in terms of $P$ and $Q$ and numerical quantities, See Equation (16) and example I.

In Fig. 1 is indicated a typical constant-voltage transmission line. If the size of conductor, or the spacing, changes at a certain point on the line, the voltage $E_{b 1}$ at that point should be marked on the diagram and calculated in the usual way. Voltages in the calculation are considered measured to neutral, and currents are in amperes per conductor. The system is considered to be three-phase.

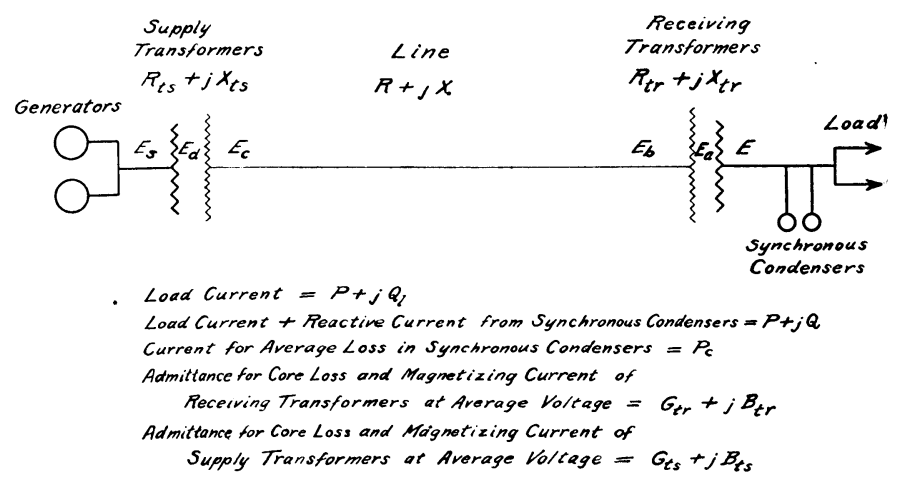

Fig. 1-Scheme of Connections of Constant-Voltage Transmission Line

Numerical values, except for $P$ and $Q$, are to be inserted in the following equations;

Current in secondary of receiving transformers

$I_{a}=P+j Q+P_{c} \quad$ amperes per conductor

Voltage induced in receiving transformers

$E_{a}=E+1 / 2 I_{a}\left(R_{t r}+j X_{t r}\right) \quad$ volts to neutral

Current in primary of receiving transformers

$I_{b}=I_{a}+E_{a}\left(G_{t r}+j B_{t r}\right)$

amperes per conductor

Voltage at receiving end of transmission line

$E_{b}=E_{a}+1 / 2 I_{b}\left(R_{t r}+j X_{t r}\right) \quad$ volts to neutral

Voltage at supply end of transmission line

$$
\begin{gathered}
E_{c}=E_{b}\left(1+\frac{Y Z}{2}+\frac{Y^{2} Z^{2}}{2 \times 3 \times 4}+\ldots\right) \\
+I_{b} Z\left(1+\frac{Y Z}{2 \times 3}+\frac{Y^{2} Z^{2}}{2 \times 3 \times 4 \times 5}\right. \\
+\ldots) \quad \text { volts to neutral }
\end{gathered}
$$

Current at supply end of transmission line

$$
\begin{array}{r}
I_{c}=I_{b}\left(1+\frac{Y Z}{2}+\frac{Y^{2} Z^{2}}{2 \times 3 \times 4}+\ldots\right) \\
+E_{b} Y\left(1+\frac{Y Z}{2 \times 3}+\frac{Y^{2} Z^{2}}{2 \times 3 \times 4 \times 5}\right. \\
+\ldots) \quad \text { amperes per conductor }
\end{array}
$$

The equations for the voltage $E_{b 1}$, and current $I_{b 1}$, etc., at an intermediate point or points where the line characteristics change, are of the same form as (5) and (6).

Note that $Y=G+j B$, the admittance of the line, (7) and $\quad Z=R+j X$, the impedance of the line, (8) The series in $Y Z$ are very convergent at commerical 
frequencies and can be quickly evaluated. It may be noted that

$$
\begin{array}{r}
1+\frac{Y Z}{2}+\frac{Y^{2} Z^{2}}{2 \times 3 \times 4}+\ldots . \\
=\cosh \sqrt{Y Z}
\end{array}
$$

$$
\text { and } \begin{aligned}
1+\frac{Y Z}{2 \times 3}+\frac{Y^{2} Z^{2}}{2 \times 3 \times 4 \times 5}+\ldots \\
=\frac{\sinh \sqrt{Y Z}}{\sqrt{Y Z}}
\end{aligned}
$$

Current in secondary of supply transformers $=I_{c}$ Voltage induced in supply transformers

$$
E_{d}=E_{c}+1 / 2 I_{c}\left(R_{t s}+j X_{t s}\right)
$$

volts to neutral

Current in primary of supply transformers

$$
I_{d}=I_{c}+E_{d}\left(G_{t s}+j B_{t s}\right)
$$

Voltage at generator terminals

$$
\text { amperes per conductor }
$$

$$
\begin{array}{r}
E_{s}=E_{d}+1 / 2 I_{d}\left(R_{t s}+j X_{t s}\right) \\
\text { volts to neutral }
\end{array}
$$

Current at generator terminals

$C+j D=I_{d}$ amperes per conductor (15)

The voltage and current at the generator terminals may thus be found in terms of $P, Q$ and numerical quantities. It is noteworthy that no trigonometrical calculations are required, but only the multiplying of complex quantitites, for which a slide rule is sufficient. The voltage $E$ remains the reference vector throughout the entire calculation.

Let $E_{s}=E^{\prime}+j E^{\prime \prime}+(P+j Q)\left(R^{\prime}+j X^{\prime}\right)$ volts to neutral

where numerical values have been found for the letters with dashes, from (14).

Equation (16) is of exactly the same form as the equation for a transmission line alone, not including transformers. A circle diagram may therefore be drawn for the complete transmission system indicated in Fig. 1, in which $E_{s}$ and $E$ are voltages kept constant by means of synchronous condensers. Such a diagram will indicate the $\mathrm{kv}-\mathrm{a}$. required from the condensers for any given load.

If, as is sometimes done, the voltage at the supply end is kept constant on the high tension side of the stepup transformers, $E_{s}$, the constant supply voltage, would be in the place occupied by $E_{c}$, Fig. 1 .

From equation (16) the absolute value of the supply voltage $E_{s}$ may be obtained. Thus,

$$
E_{\text {。 }}{ }^{2}=\left(E^{\prime}+P R^{\prime}-Q X^{\prime}\right)^{2}+\left(E^{\prime \prime}+P X^{\prime}+Q R^{\prime}\right)^{2}
$$

In the case of a constant-voltage line, $P$ and $Q$ are the only variables.

Equation (17) is the equation of a circle. It reduces to

$$
\begin{aligned}
\left(P+\frac{E^{\prime} R^{\prime}+E^{\prime \prime} X^{\prime}}{R^{\prime 2}+X^{\prime 2}}\right)^{2} & +\left(Q-\frac{E^{\prime} X^{\prime}-E^{\prime \prime} R^{\prime}}{R^{\prime 2}+X^{\prime 2}}\right)^{2} \\
& -\frac{E_{s}{ }^{2}}{R^{\prime 2}+X^{\prime 2}}=0
\end{aligned}
$$

Since $P$ and $Q$ represent currents, equation (18) should be multiplied throughout by

$$
\frac{3 E}{1000}
$$

to give a circle showing the relation between $\mathrm{kw}$. and reactive kv-a. at the receiver end of the constantvoltage line.

The center of the circle is the point $\left(a^{\prime}, b^{\prime}\right)$ where

$$
\begin{aligned}
a^{\prime}=-\frac{3 E}{1000} & \frac{E^{\prime} R^{\prime}+E^{\prime \prime} X^{\prime}}{R^{\prime 2}+X^{\prime 2}} \quad \mathrm{kw} . \\
b^{\prime}=+\frac{3 E}{1000} & \frac{E^{\prime} X^{\prime}-E^{\prime \prime} R^{\prime}}{R^{\prime 2}+X^{\prime 2}} \quad \mathrm{kv}-\mathrm{a} .
\end{aligned}
$$

The radius is

$$
c^{\prime}=+\frac{3 E}{1000} \quad \frac{E_{s}}{\sqrt{R^{\prime 2}+X^{\prime 2}}} \quad \mathrm{kv}-\mathrm{a} .
$$

In order to plot the reactive $\mathrm{kv}$-a. required from the synchronous condensers, first draw a straight line at

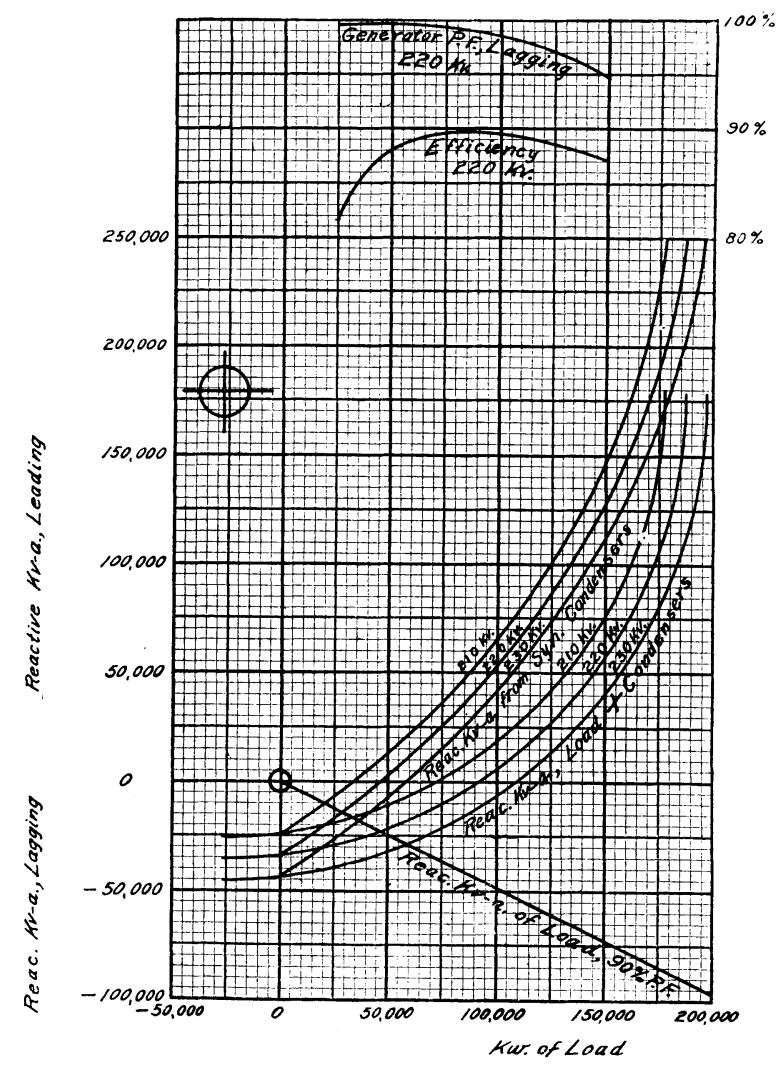

Fig. 2-Circle Diagram for 220,000-Volt, ConstantVoltage Transmission Line (See example 1)

an angle $\theta$ below the base line, where $\cos \theta$ is the power factor lagging, of the load. If the power factor is not the same at all loads, the line will not be straight, but will be a curve showing the reactive $\mathrm{kv}$-a. of the load from no load to full load. By means of a pair of dividers add the reactive kv-a. of the load to the corresponding ordinate of the circle, thus plotting the curve of kv-a. required from the synchronous condensers. See Fig. 2. 
Theoretical Limit of Load, in Kilowatts

Maximum Load $=c^{\prime}+a^{\prime} \quad \mathrm{kw}$.

This is numerically less than $c^{\prime}$ since $a^{\prime}$ is a negative quantity. It may read from the circle diagram as it is the farthest distance to the right reached by the circle.

Calculated Value of Reactive $K v-a$. The method described in this article is not necessarily a graphical method. It is possible to calculate the reactive kv-a. directly, which is sometimes desirable in order to obtain a more precise result than that obtained graphically from the diagram. A direct calculation made in this way is less work than a "trial and error" method, which would generally involve calculating the problem more than once.

The value of the reactive $\mathrm{kv-a.,} \frac{3 E Q}{1000}$, for a given power load $\frac{3 E P}{1000}$, may be found from the following equation:

$$
\left(b^{\prime}-\frac{3 E Q}{1000}\right)^{2}=c^{\prime}-\left(\frac{3 E P}{1000}-a^{\prime}\right)^{2}
$$

The reactive $\mathrm{kv}-\mathrm{a}$. required from the synchronous condensers are equal to

$$
\frac{3 E Q}{1000}+\frac{3 E P}{1000} \frac{\sin \theta}{\cos \theta} \quad \mathrm{kv}-\mathrm{a} \text {. }
$$

where the power load is $\frac{3 E P}{1000} \mathrm{kw}$. at a lagging power

factor $\cos \theta$. It should be remembered that $b^{\prime}$ is a positive quantity and $a^{\prime}$ is a negative quantity. It is worth while checking the results of equations (24) and (25) by drawing the circle diagram and obtaining the same results graphically.

Concentric Circles. Since $a^{\prime}$ and $b^{\prime}$ which give the center, are independent of the constant supply voltage $E_{s}$, and since the radius $c^{\prime}$ is directly proportional to $E_{s}$, it is evident that a number of circles corresponding to different values of $E_{s}$ may be drawn about the same center. See Fig. 2.

Total Losses.

Let $A=E^{\prime}+P R^{\prime}-Q X^{\prime} \quad$ volts to neutral and $B=E^{\prime \prime}+P X^{\prime}+Q R^{\prime}$ volts to neutral

The losses in the transmission system equal

$$
\frac{3}{1000}(A C+B D-E P) \quad \mathrm{kw} \text {. }
$$

This does not include the generator osses. When the constant voltage $E_{s}$ is on the high tension side of the step-up transformers the losses in the step-up transformers are not included in expression (28). As mentioned before, an average value was assumed for the transformer core loss and the condenser loss. The quantities $C$ and $D$ are found from equation (15).

Efficiency of the Transmission System.

$$
\text { Efficiency }=\frac{100 E P}{A C+B D} \quad \text { per cent }
$$

$K w$. at supply end

$$
\frac{3}{1000}(A C+B D) \quad \text { kw. }
$$

$K v-a$. at supply end

$$
\frac{3 E_{s} \sqrt{C^{2}+D^{2}}}{1000} \quad \mathrm{kv}-\mathrm{a} .
$$

Power factor at supply end

$$
\frac{100(A C+B D)}{E_{s} \sqrt{C^{2}+D^{2}}} \quad \text { per cent }
$$

The "supply end" is the point where the voltage $E$, is kept constant. If this at the generator terminals, as indicated in Fig. 1, expression (32) gives the power factor of the generator load. Whether this is leading or lagging must be determined from the following expression:

Reactive kv-a. at supply end

$$
\frac{3}{1000}(A D-B C) \quad \text { kv-a. }
$$

When this quantity is positive the reactive kv-a. and the power factor are leading, and when it is negative, they are lagging.

\section{EXAMPLE I}

Length of line $=200$ miles

Frequency $=60$ cycles

$R+j X=23.2+j 160 \mathrm{ohms}$

$Y=+j 0.00106 \mathrm{mho}$

$1+\frac{Y Z}{2}+\ldots=0.91637+j 0.01195$

$1+\frac{Y Z}{2.3}+\ldots=0.97197+j 0.00403$

$P_{c}=8.66$ amperes

$R_{t r}+j X_{t r}=1.33+j 24.0 \mathrm{ohms}$

$G_{t r}+j B_{\text {tr }}=0.000,022,5-j 0.000,187,5 \mathrm{mho}$

$R_{t s}+j X_{t s}=1.61+j 29.0 \mathrm{ohms}$

$G_{t s}+j B_{t s}=0.000,018,6-j 0.000,155,0 \mathrm{mho}$

$E=115,470$ volts to neutral (line voltage 200,000)

$I_{a}=P+j Q+8.7$

$E_{a}=115,480+j 100+(P+j Q)(0.7+j 12.0)$

$I_{b}=(P+j Q)(1.002+j 0.0002)+11.3-j 21.6$

$E_{b}=115,740+j 220+(P+j Q)(1.3+j 24.0)$

$E_{c}=109,680+j 2870+(P+j Q)(22.9+j 178.0)$

$I_{c}=(P+j Q)(0.894+j 0.013)+9.9+j 99.5$

$E_{d}=108,240+j 3090+(P+j Q)(23.4+j 191.0)$

$I_{d}=(P+j Q)(0.924+j 0.013)+12.4+j 82.8$

$E_{\mathrm{s}}=107,050+j 3340+(P+j Q)(23.9+j 204.4)$

$=E^{\prime}+j E^{\prime \prime}+(P+j Q)\left(R^{\prime}+j X^{\prime}\right)$

$a^{\prime}=-26,600 \mathrm{kw}$.

$b^{\prime}=178,300 \mathrm{kv}-\mathrm{a}$.

$c^{\prime}=213,800 \mathrm{kv}-\mathrm{a}$.

when $E_{s}=127,020$ volts to neutral (line voltage $=220,000$ ). See Fig. 2, which shows the desired characteristics of the system. 